\title{
Development of low-background photomultiplier tubes for liquid xenon detectors
}

\section{XMASS Collaboration}

\author{
K. Abe, ${ }^{a, e}$ Y. Chen, ${ }^{a}$ K. Hiraide, ${ }^{a, e}$ K. Ichimura,,${ }^{a, e, 1}$ S. Imaizumi, ${ }^{a}$ N. Kato, ${ }^{a}$ Y. Kishimoto, ${ }^{a, 1, e}$
}

K. Kobayashi, ${ }^{a, e, 2}$ M. Kobayashi, ${ }^{a, 3}$ S. Moriyama, ${ }^{a, e}$ M. Nakahata, ${ }^{a, e}$ K. Sato, ${ }^{a, 4}$ H. Sekiya, ${ }^{a, e}$

T. Suzuki, ${ }^{a}$ A. Takeda, ${ }^{a, e}$ S. Tasaka,,${ }^{a, 5}$ M. Yamashita, ${ }^{a, e, 4}$ B. S. Yang, ${ }^{b}$ N. Y. Kim, ${ }^{c}$ Y. D. Kim, ${ }^{c}$

Y. H. Kim, ${ }^{c, h}$ R. Ishii, ${ }^{d}$ Y. Itow, ${ }^{d, f}$ K. Kanzawa, ${ }^{d}$ K. Masuda, ${ }^{d}$ K. Martens, ${ }^{e}$ A. Mason,,${ }^{e, 6}$

Y. Suzuki, ${ }^{e}{ }^{7}$ K. Miuchi, ${ }^{g}$ Y. Takeuchi, ${ }^{g, e}$ K. B. Lee, ${ }^{h}$ M. K. Lee, ${ }^{h}$ Y. Fukuda, ${ }^{i}$ H. Ogawa, ${ }^{j, e}$

K. Nishijima, ${ }^{k}$ K. Fushimi, ${ }^{l}$ B. D. Xu, ${ }^{m, e}$ S. Nakamura ${ }^{n}$

${ }^{a}$ Kamioka Observatory, Institute for Cosmic Ray Research, the University of Tokyo, Higashi-Mozumi, Kamioka, Hida, Gifu 506-1205, Japan

${ }^{b}$ Center for Axion and Precision Physics Research, Institute for Basic Science, Daejeon 34051, South Korea

${ }^{c}$ Center for Underground Physics, Institute for Basic Science, 70 Yuseong-daero 1689-gil, Yuseong-gu, Daejeon 305-811, South Korea

${ }^{d}$ Institute for Space-Earth Environmental Research, Nagoya University, Nagoya, Aichi 464-8601, Japan

${ }^{e}$ Kavli Institute for the Physics and Mathematics of the Universe (WPI), the University of Tokyo, Kashiwa, Chiba 277-8582, Japan

${ }^{f}$ Kobayashi-Maskawa Institute for the Origin of Particles and the Universe, Nagoya University, Furo-cho, Chikusa-ku, Nagoya, Aichi 464-8602, Japan

${ }^{g}$ Department of Physics, Kobe University, Kobe, Hyogo 657-8501, Japan

${ }^{h}$ Korea Research Institute of Standards and Science, Daejeon 305-340, South Korea

${ }^{i}$ Department of Physics, Miyagi University of Education, Sendai, Miyagi 980-0845, Japan

${ }^{j}$ Department of Physics, College of Science and Technology, Nihon University, Kanda, Chiyoda-ku, Tokyo 101-8308, Japan

${ }^{k}$ Department of Physics, Tokai University, Hiratsuka, Kanagawa 259-1292, Japan

${ }^{l}$ Department of Physics, Tokushima University, 2-1 Minami Josanjimacho Tokushima city, Tokushima 7708506, Japan

${ }^{m}$ Department of Engineering Physics, Tsinghua University, Haidian District, Beijing, China 100084

${ }^{n}$ Department of Physics, Faculty of Engineering, Yokohama National University, Yokohama, Kanagawa 240-8501, Japan

E-mail: xmass.publications16@km.icrr.u-tokyo.ac.jp

\footnotetext{
${ }^{1}$ Now at Research Center for Neutrino Science, Tohoku University, Sendai 980-8578, Japan

${ }^{2}$ Now at Waseda Research Institute for Science and Engineering, Waseda University, Shinjuku, Tokyo 169-8555,

${ }^{3}$ Now at Physics Department, Columbia University, New York, NY 10027, USA

${ }^{4}$ Now at Institute for Space-Earth Environmental Research, Nagoya University, Nagoya, Aichi 464-8601, Japan

${ }^{5}$ Now at Gifu University, Gifu 501-1193, Japan

${ }^{6}$ Now at Department of Physics, University of Oxford, Oxford, Oxfordshire, United Kingdom

${ }^{7}$ Now at Institute for Cosmic Ray Research, the University of Tokyo, Kashiwa, Chiba 277-8582, Japan
} Japan 
Aвstract: We successfully developed a new photomultiplier tube (PMT) with a three-inch diameter, convex-shaped photocathode, R13111. Its prominent features include good performance and ultra-low radioactivity. The convex-shaped photocathode realized a large photon acceptance and good timing resolution. Low radioactivity was achieved by three factors: (1) the glass material was synthesized using low-radioactive-contamination material; (2) the photocathode was produced with ${ }^{39} \mathrm{~K}$-enriched potassium; and (3) the purest grade of aluminum material was used for the vacuum seal. As a result each R13111 PMT contains only about $0.4 \mathrm{mBq}$ of ${ }^{226} \mathrm{Ra}$, less than $2 \mathrm{mBq}$ of ${ }^{238} \mathrm{U}, 0.3 \mathrm{mBq}$ of ${ }^{228} \mathrm{Ra}, 2 \mathrm{mBq}$ of ${ }^{40} \mathrm{~K}$ and $0.2 \mathrm{mBq}$ of ${ }^{60} \mathrm{Co}$. We also examined and resolved the intrinsic leakage of Xe gas into PMTs that was observed in several older models.

We thus succeeded in developing a PMT that has low background, large angular acceptance with high collection efficiency, good timing resolution, and long-term stable operation. These features are highly desirable for experiments searching for rare events beyond the standard model, such as dark matter particle interactions and neutrinoless double beta decay events.

Keywords: Noble liquid detectors (scintillation, ionization, double-phase), Photon detectors for UV, visible and IR photons (solid-state) (PIN diodes, APDs, Si-PMTs, G-APDs, CCDs, EBCCDs, EMCCDs, CMOS imagers, etc)

ARXiv EPrint: 1234.56789 


\section{Contents}

1 Introduction 1

2 The R13111 PMT 2

2.1 The structure of the PMT 2

2.2 Performance of the PMT 3

3 Reduction of RI $\quad 4$

3.1 Methods used in our RI measurements 4

3.2 Key items for RI reduction $\quad 6$

3.2.1 The glass beads $\quad 6$

3.2.2 The potassium compound used for photocathode production 7

$\begin{array}{llr}3.2 .3 & \text { The aluminum seal } & 8\end{array}$

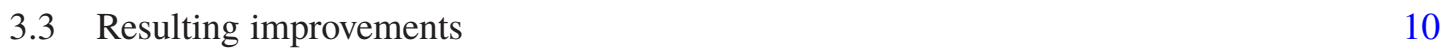

$\begin{array}{lll}\text { 3.3.1 RIs of the PMT components } & 10\end{array}$

$\begin{array}{lll}\text { 3.3.2 RIs of fully assembled PMTs } & 13\end{array}$

$\begin{array}{lll}\text { 3.3.3 Comparison to other PMT used for LXe detectors } & 14\end{array}$

4 Afterpulse study related to the Xe leak problem $\quad 15$

$\begin{array}{lll}4.1 & \text { Identification of the leaking component } & 15\end{array}$

$\begin{array}{lll}4.2 & \text { Setup of the study } & 16\end{array}$

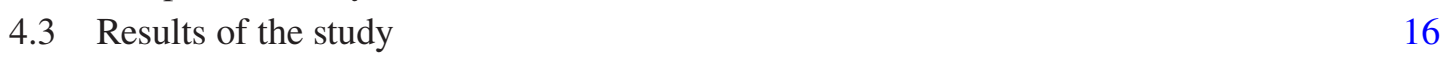

$\begin{array}{llr}5 & \text { Conclusion } & 17\end{array}$

$\begin{array}{llr}6 & \text { Acknowledgments } & 18\end{array}$

\section{Introduction}

In modern astroparticle physics, one of the foremost scientific challenges is to directly detect the dark matter interactions with detector materials. Weakly Interacting Massive Particles (WIMPs) are one of the leading dark matter candidates and they would produce a signal via elastic scattering on a target nucleus. Liquid xenon (LXe) is widely used for dark matter searches, owing to its well-suited material properties for low-background (BG) searches, such as high light yield, high density, good stopping power. Many experiments have reported results of dark matter searches using LXe [1-4].

The expected signal rate for LXe dark matter experiments currently is known to be at most one event/ton/year. Since the amount of BG directly impacts the sensitivity of any dark matter search, the BG event rate from the detector components must be as low as possible. With proper shielding against external radiation, radioactive impurities (RIs) in the detector materials have 
generally becomes the most important source of BG in direct-detection dark matter experiments. Such experiments typically require a large number of photomultiplier tubes (PMTs), which often are the largest contributor to the RI budget in a detector. This was also the case for the XMASS-I experiment, which carried out many rare-event searches including searches for WIMP dark matter [5-10]. The R10789 PMTs were the one of main BG sources in the experiment, even though they had been specifically developed for the XMASS-I experiment [11].

New types of sensors, such as silicon photomultipliers, are being rapidly developed, however the PMT still has the main role for upcoming experiments such as XENONnT [4] and LZ [12]. To achieve higher sensitivity in future larger LXe detectors, it is crucial to develop PMTs with still lower BG; lower RIs and better performance. Even after all the effort to date, including the R10789 development, this is still true. Such improved PMTs will not only be needed to improve dark matter searches, but also be useful in other rare event searches beyond the standard model such as search for neutrinoless double beta decay. Based on the R10789 PMTs used in the XMASS-I experiment, we developed the new R13111 low-BG PMT with good performance and ultra-low RI contamination. To reduce the RIs, we screened in particular those parts that contributed most to the total RI of the R10789 PMT.

We adopted a convex-shaped photocathode for the detection of photons incident on the photocathode from side direction of the PMT. When a PMT array is used in a single-phase liquid noble gas detector, this allows effective identification of surface BG events by detecting scintillation photons from the surface on which the PMTs are arranged. This property is of particular interest for us, since the R10789 with its flat photocathode was not suited for the identification of BG events at these surface. The surface BG was a very significant BG in XMASS-I [8].

A problem with Xe leakage and the corresponding Xe afterpulses were reported in $[13,14]$ for the R11410 Hamamatsu low-RI PMTs employed in recent LXe detectors [3, 15]. We also carried out a detailed study of this leakage problem. In addition to the fact that the afterpulses are adding BG, a large Xe leak ultimately makes the PMT inoperable. Since the R11410 PMT has many structures in common with other Hamamatsu low-BG PMT, Xe leakage is a problem that needs to be solved for the long-term stability of all these low-BG PMTs.

In the following section, we discuss the structure and performance of the R13111. In section 3, we explain the RI reduction in the PMT, including our methods for RI measurements, key items for RI reduction, and report the results. We also discuss the ultra-low BG, high-purity germanium (HPGe) detectors used to measure the RI of the assembled PMT with high sensitivity in section 3. We describe the Xe leak study in section 4. Our conclusions are given in section 5.

\section{The R13111 PMT}

\subsection{The structure of the PMT}

A picture of an R13111 PMT is shown in Figure 1. Figure 2 shows a schematic view of this R13111 PMT. We designed it based on the R10789, which has a smaller diameter of two inches. Considering this PMT's potential for use in future large-scale detectors, we adopted a three inch diameter for the R13111. As with other PMTs used in LXe detectors, the R13111 PMT uses a quartz window, which is low in RIs and has high transmittivity for the VUV light from LXe scintillation. Table 1 lists all 


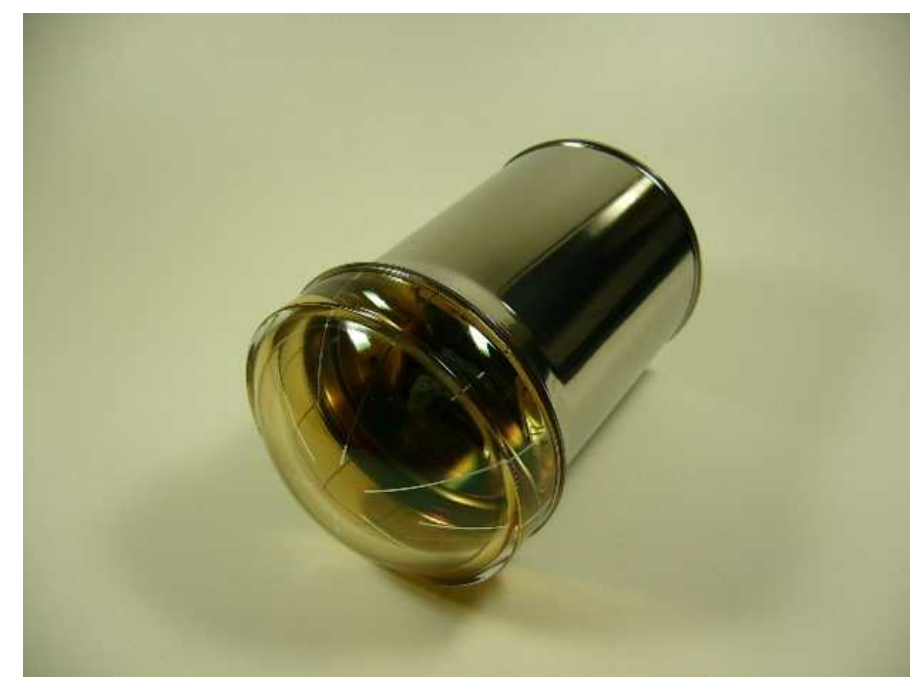

Figure 1. R13111

the PMT parts with their respective weight contribution to one R13111 PMT; their locations in the PMT structure can be seen in Figure 2. A quartz spacer is used to support the dynode structure. The disk feedthrough is a small feedthrough including the glass part used to feed current through the disk electrode. A screw, nut, and washer attach this disk feedthrough to the disk electrode. A lead wire and ribbons provide connections between the electrodes and the stem pins at the back of the PMT. A spring is used to connect the cathode to the PMT body.

We adopted a convex-concave shape for the R13111 window. The photocathode is deposited on the window's inside concave surface. Because of this photocathode geometry, the R13111 PMT has a large angular acceptance. This is a main structural difference to the R10789, which has a flat photocathode. In addition to this photocathode shape, high collection efficiency for the whole photocathode region was achieved by designing the geometry of the body parts and the dynodes using photoelectron-tracking simulations conducted by the manufacturer. The design collection efficiency is more than $90 \%$ for $97 \%$ of the total photocathode area.

The convex geometry of the photocathode also improves the timing resolution, since this geometry makes the photoelectron track length more uniform over the photocathode area. The design of the PMT body and its dynodes also are optimized for timing resolution. This improvement enables better particle identification through the decay time of the scintillation signal, which directly translates into better BG suppression.

\subsection{Performance of the PMT}

The acceptance, collection efficiency and timing resolution of the R13111 PMT, were measured with the methods described in [20].

At room temperature, a total of 11 PMTs were measured. The collection efficiency times the quantum efficiency was examined and evaluated relative to the center of the photocathode. Observed relative efficiencies were above $70 \%$ at all test points [20]. Furthermore, we evaluated the transit time spread (TTS). The TTS for the whole photocathode area was measured using diffused $405 \mathrm{~nm}$ laser light with $63 \mathrm{psec}$ pulse width (FWHM) on three of the R13111 PMT. The measured 


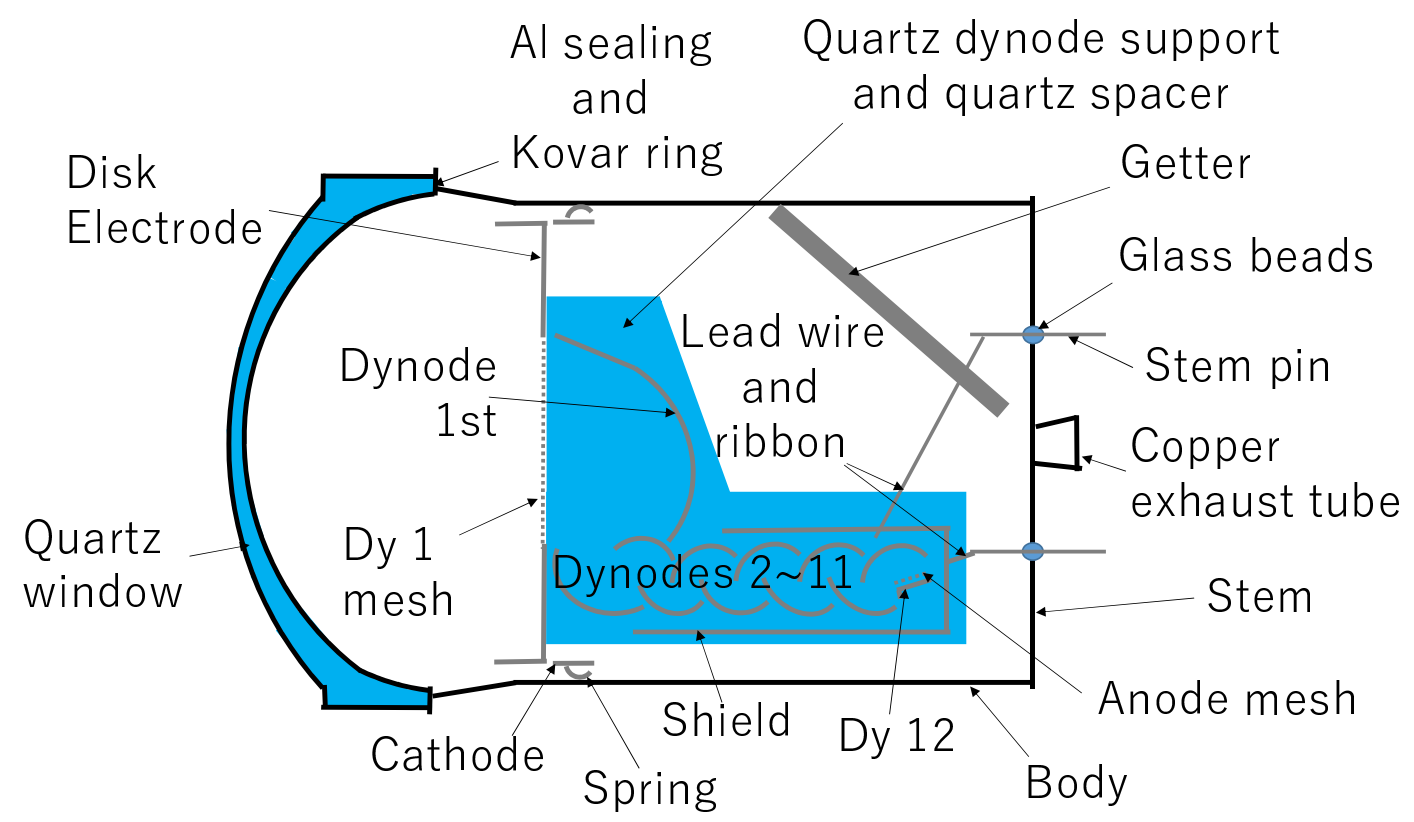

Figure 2. Schematic view of R13111. The disk stopper, the disk feedthrough, the number plate, the screws, the nut and the washer are attached to the disk electrode.

TTS (FWHM) from the three PMTs was $2.11_{-0.18}^{+0.31}$ nsec. Considering that TTS becomes shorter at longer incident wavelength, this number is consistent with the design value, $2.9 \mathrm{nsec}$ for $175 \mathrm{~nm}$, the Xe scintillation wavelength. This constitutes a significant improvement over the R10789's TTS of $6.87 \pm 0.31 \mathrm{nsec}$, which was obtained using the same setup.

\section{Reduction of RI}

In this section, we report the achieved reduction of RI. We first describe the methods used for the RI measurements. Next we discuss the key materials targeted for RI reduction. We also report the RIs for these individual materials as well as for the whole assembled PMT. Finally we summarize the measured RIs and compare them to those reported for other PMTs.

\subsection{Methods used in our RI measurements}

To measure the radioactivity of materials we used four different low BG HPGe detectors manufactured by CANBERRA and installed underground at Kamioka. We also employed two different mass-spectrometry methods; inductively coupled plasma mass spectrometry (ICP-MS) and glowdischarge mass spectrometry (GD-MS). Three of the HPGe detectors are coaxial p-type devices. The other is a coaxial n-type. The crystal masses and efficiencies of these HPGe detectors are about $2.5 \mathrm{~kg} / 120 \%$ (p-type, GC series), $1.9 \mathrm{~kg} / 100 \%$ (p-type GC series), $1.7 \mathrm{~kg} / 80 \%$ (p-type, T2FA series), and $1.9 \mathrm{~kg} / 100 \%$ (n-type GR series) ${ }^{1}$. Details of the HPGe detectors (except for the T2FA series HPGe detector, which we describe below), the ICP-MS, and the GD-MS are provided in [11].

\footnotetext{
${ }^{1}$ The efficiency is given relative to the efficiency of a three-inch diameter three-inch thick $\mathrm{NaI}(\mathrm{Tl})$ for $1.332 \mathrm{MeV}$ gamma-ray from ${ }^{60} \mathrm{Co}$ source which is positioned $25 \mathrm{~cm}$ away from the detector.
} 
Table 1. List of R13111 parts, including their respective weight per PMT. The ratio of the weight used for the HPGe measurements to the weight per PMT is also shown.

\begin{tabular}{|c|c|c|c|c|}
\hline Samples & Materials & $\begin{array}{l}\text { Weight for } \\
\text { HPGe (g) }\end{array}$ & $\begin{array}{r}\text { Weight per } \\
\text { PMT (g) }\end{array}$ & Ratio \\
\hline Body & Co free metal & 4450 & 84.9 & 52.4 \\
\hline Stem & Co free metal & 3557 & 62 & 57.4 \\
\hline Stem pin & Kovar alloy & 92 & 1.6 & 58 \\
\hline Glass beads & Made from high-purity glass & 186 & 2.3 & 80.8 \\
\hline Quartz window & Synthetic quartz & 2610 & 82.1 & 31.8 \\
\hline Quartz dynode support & Synthetic quartz & 360 & 11.8 & 30.5 \\
\hline Quartz spacer & Synthetic quartz & 37.5 & 0.75 & 50 \\
\hline Al Seal & Aluminum & 109.6 & 0.9 & 121.8 \\
\hline Kovar ring & Kovar alloy & 196 & 3.3 & 59.4 \\
\hline Antimony for evaporation & Antimony & 1.6 & 0.022 & 72.7 \\
\hline Al for evaporation & Aluminum & 19.9 & 0.064 & 311 \\
\hline Hermetic & glass, Kovar & 103 & 0.19 & 542 \\
\hline Lead wire, Ribbons & Nickel & 189 & 1.88 & 101 \\
\hline Screw, nut, washer, getter stopper & Non-Magnetic (NM) stainless steel & 115 & 1.2 & 95.8 \\
\hline Cathode & NM stainless steel $(0.25 \mathrm{t})$ & 133.5 & 2.4 & 55.6 \\
\hline Dynode 1st stage & $\mathrm{NM}$ stainless steel $(0.3 \mathrm{t})$ & 212 & 5.1 & 41.6 \\
\hline Dynode (2nd to 12 th stage) & $\mathrm{NM}$ stainless steel $(0.2 \mathrm{t})$ & 375 & 7.5 & 50 \\
\hline Shield & $\mathrm{NM}$ stainless steel $(0.3 \mathrm{t})$ & 331 & 6.6 & 50.1 \\
\hline Spring, number plate & NM stainless steel & 32.4 & 0.34 & 95.3 \\
\hline Anode mesh & NM stainless steel $(0.25 \mathrm{t})$ & 15.6 & 0.1 & 156 \\
\hline Disk stopper & NM stainless steel $(0.18 \mathrm{t})$ & 41.4 & 0.87 & 47.6 \\
\hline Dynode 1st stage mesh & SUS304 (0.05 t) & 8.1 & 0.16 & 50.6 \\
\hline Disk & $\mathrm{NM}$ stainless steel $(0.5 \mathrm{t})$ & 654 & 12.6 & 51.9 \\
\hline Getter & Zr-Al alloy & 7.0 & 0.07 & 100 \\
\hline Exhaust tube & Copper & 86 & 1.4 & 60 \\
\hline
\end{tabular}

To measure low radioactivity with high sensitivity, especially for fully assembled PMTs, we installed a T2FA series HPGe detector in 2016. This detector was manufactured by CANBERRA France. At Kamioka it is located at the center of a $23 \times 23 \times 48 \mathrm{~cm}$ sample chamber. To shield it from environmental gamma-rays, this chamber is surrounded by $1 \mathrm{~cm}$ of $6 \mathrm{~N}$ grade copper, $5 \mathrm{~cm}$ of OFHC copper, a $2.5 \mathrm{~cm}$ lead layer with a ${ }^{210} \mathrm{~Pb}$ activity of only $5 \mathrm{~Bq} / \mathrm{kg}$, and a $20 \mathrm{~cm}$ lead layer with a ${ }^{210} \mathrm{~Pb}$ activity of $140 \mathrm{~Bq} / \mathrm{kg}$. Like for the other three Kamioka HPGe detectors, this sample chamber is continually flushed with Rn-free air in which the ${ }^{222} \mathrm{Rn}$ concentration was less than a few tens of $\mathrm{mBq} / \mathrm{m}^{3}$ in order to suppress radon diffusion into the chamber. The sample space in this detector is large enough to measure 13 PMTs at once, while our GC type HPGe detectors can only hold 2 PMTs. The signal from this HPGe detector was fed into an ORTEC 572A main amplifier. The amplified signal was recorded by a CAEN DT5724 waveform digitizer with a $100 \mathrm{MHz}$ sampling rate, which on another channel also recorded the same signal without amplification. The maximum pulse height is used to estimate the deposited energy. Noise can be rejected based on pulse shape. Detector calibrations were frequently performed with radioactive sources of ${ }^{60} \mathrm{Co},{ }^{133} \mathrm{Ba}$, and ${ }^{137} \mathrm{Cs}$. In order to evaluate detection efficiencies, we performed Geant4 [16] based Monte Carlo simulations. 


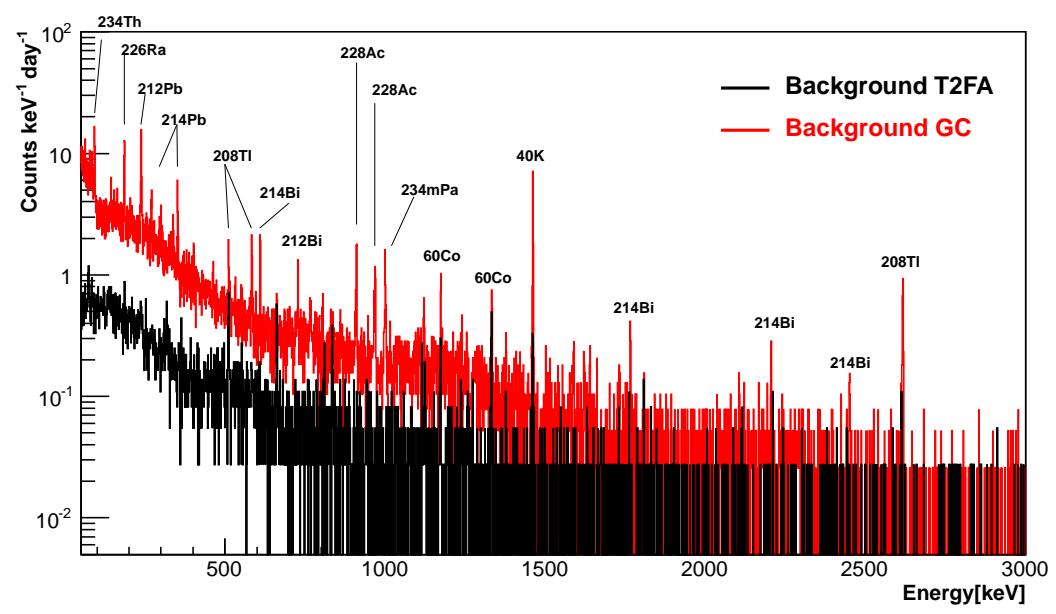

Figure 3. BG spectrum of the T2FA p-type HPGe detector (36 live days, black), together with the BG spectrum of a GC p-type HPGe detector (38 live days, red). The bin width is $1 \mathrm{keV}$.

Similar to our procedure for the other HPGe detectors reported in [11], we estimated systematic uncertainties for detection efficiencies through comparison between calibration data and simulation to be $+30 \%$ and $-10 \%$. Since the systematic error is common to all measurements, the results in the following sections are given only with their respective statistical errors. Figure 3 shows the $\mathrm{BG}$ spectrum of this new detector together with one from one of the other coaxial p-type HPGe detectors in Kamioka. Thanks to its ultra-low BG and its large sample chamber, the sensitivity to ${ }^{226} \mathrm{Ra}$ and ${ }^{228} \mathrm{Ra}$ are improved from $O\left(10^{3}\right) \mu \mathrm{Bq} / \mathrm{PMT}$ for the GC type Ge detectors to $O\left(10^{2}\right)$ $\mu \mathrm{Bq} / \mathrm{PMT}$ for the new T2FA type Ge detector.

GD-MS and ICP-MS measurements were carried out by Eurofins EAG Materials Science Tokyo Corporation[17] and Toray Research Center, Inc.[18]. The systematic uncertainties caused by processes such as weighing, pretreatment and the measurement itself are estimated as $\pm 30 \%$ for GD-MS results and $\pm 10 \%$ for ICP-MS results from standard samples and repeated measurements.

\subsection{Key items for RI reduction}

We concentrated our efforts at RI reduction on those items that contributed most to the radioactivity of the R10789 PMT[11]: the glass beads used to seal the electrical feedthroughs in the base of the PMT, the potassium compound used in the photocathode production, and the aluminum seal for the entrance window. As a result of this effort we achieved a significantly lower RI contamination for the new R13111 PMT.

\subsubsection{The glass beads}

Glass beads are used as electrical insulation for the feedthroughs (Figure 2), and they contributed about $70 \%$ of the total ${ }^{226} \mathrm{Ra}$ activity in the R10789 PMT. Since the total amount of glass needed is not large ( $2 \mathrm{~g} / \mathrm{PMT}$ as shown in Table 1$)$ we decided that the glass beads should be made from high-purity reagents. The new glass was synthesized with the cooperation of OKAMOTO GLASS Co, Ltd. We determined the required reagents and their amounts by analyzing the glass beads used 
for the R10789, obtaining the results listed in Table 2. High purity reagents were obtained from Kojundo Chemical Laboratory Ltd. After checking the RIs of all reagents, the synthesis and milling processes were carried out by OKAMOTO GLASS Co, Ltd. Then, the glass powder was molded and melted into glass beads by the manufacturer.

Table 2 shows the amount and measured RI for each reagent, the synthesized glass, the glass after the milling process, and the glass beads, together with the measurements for the glass beads used in the R10789. Compared to the R10789 glass beads, the RIs of the newly synthesized glass beads are reduced to less than $2 \%$, less than $22 \%$, less than $7 \%$ and $12 \%$ for ${ }^{226} \mathrm{Ra},{ }^{238} \mathrm{U},{ }^{232} \mathrm{Th}$ and ${ }^{40} \mathrm{~K}$, respectively. We measured the RIs of the glass just after synthesis as well as of the milled powder in order to check for any unexpected contamination introduced in individual processing steps. We observed no significant contamination or increase above expectation from the reagents used to synthesize the glass. The synthesized glass shows smaller amounts of ${ }^{226} \mathrm{Ra}$ than the sum of the reagents, though the measurements could be considered compatible given their uncertainties. Since the yield of the synthesis was not $100 \%$, we cannot deny the possibility of such a reduction occurring during the synthesis process. The RIs of the glass after milling are higher than those of the glass after synthesis. We speculate that there was contamination from the air or from the milling tools during the milling process. The milling process uses ceramic tools (ceramic balls and a ceramic pot). An ordinary ceramic tool we checked included $(3.76 \pm 0.02) \cdot 10^{3} \mu \mathrm{Bq} / \mathrm{g}$ of ${ }^{226} \mathrm{Ra}$, $(4.74 \pm 0.02) \cdot 10^{3} \mu \mathrm{Bq} / \mathrm{g}$ of ${ }^{228} \mathrm{Ra}$ and $(12.0 \pm 0.1) \cdot 10^{3} \mu \mathrm{Bq} / \mathrm{g}$ of ${ }^{40} \mathrm{~K}$. To lower their impact, we also prepared high-purity ceramic tools (Nikkato, alumina ball SSA-999W and alumina pot mill SSA-999W A-5). The RIs of the new tools were $(4.2 \pm 0.1) \cdot 10^{2} \mu \mathrm{Bq} / \mathrm{g}$ of ${ }^{226} \mathrm{Ra}$, $(35 \pm 3) \mu \mathrm{Bq} / \mathrm{g}$ of ${ }^{228} \mathrm{Ra}$ and $(68 \pm 22) \mu \mathrm{Bq} / \mathrm{g}$ of ${ }^{40} \mathrm{~K}$. These numbers are one to two orders of magnitude smaller than the ordinary tools, however they are still quite large compared to the glass beads themselves (see Table 2). The possibility of contamination from the tools cannot be rejected.

Table 2. Results of HPGe measurements for the glass-bead-related materials. The unit is $\mu \mathrm{Bq} / \mathrm{g}$. Errors quoted are purely statistical. See the text for the systematic error.

\begin{tabular}{|c|r|r|r|r|r|r|r|}
\hline Samples & $\begin{array}{r}\text { component } \\
\text { ratio [\%] }\end{array}$ & ${ }^{226} \mathrm{Ra}$ & $\begin{array}{r}{ }^{238} \mathrm{U} \\
\left({ }^{234} \mathrm{Th}\right)\end{array}$ & ${ }^{210} \mathrm{~Pb}$ & ${ }^{228} \mathrm{Ra}$ & \\
\hline $\mathrm{SiO}_{2} 5 \mathrm{~N}$ grade & 64 & $<11$ & $<1.5 \cdot 10^{2}$ & $<1.1 \cdot 10^{3}$ & $<13$ & $<1.5 \cdot 10^{2}$ & $<4.4$ \\
\hline $\mathrm{B}_{2} \mathrm{O}_{3}$ 4N5 Grade & 3 & $<45$ & $<1.8 \cdot 10^{2}$ & $<4.6 \cdot 10^{2}$ & $<44$ & $<4.5 \cdot 10^{2}$ & $<12$ \\
\hline $\mathrm{Na}_{2} \mathrm{~B}_{4} \mathrm{O}_{7} 4 \mathrm{~N} 5$ Grade & 24 & $(1.8 \pm 0.6) \cdot 10^{2}$ & $<3.9 \cdot 10^{2}$ & $<1.3 \cdot 10^{3}$ & $<74$ & $<1.1 \cdot 10^{3}$ & $<25$ \\
\hline $\mathrm{Al}(\mathrm{OH})_{3} 4 \mathrm{~N}$ grade & 9 & $<25$ & $<2.3 \cdot 10^{2}$ & $<1.5 \cdot 10^{3}$ & $<42$ & $<2.0 \cdot 10^{2}$ & $<7.6$ \\
\hline \hline Sum & & $36 \pm 15$ & $<1.3 \cdot 10^{2}$ & $<6.5 \cdot 10^{2}$ & $<19$ & $<2.6 \cdot 10^{2}$ & $<6.6$ \\
\hline \hline Glass after synthesis & & $12 \pm 4$ & $57 \pm 26$ & $<61$ & $<9.4$ & $(1.5 \pm 0.5) \cdot 10^{2}$ & $<2.5$ \\
\hline Glass after milling & & $24 \pm 5$ & $<97$ & $<99$ & $17 \pm 4$ & $(1.3 \pm 0.6) \cdot 10^{2}$ & $<4.0$ \\
\hline Glass beads & & $<15$ & $<72$ & $<1.9 \cdot 10^{2}$ & $<9.7$ & $(1.7 \pm 0.7) \cdot 10^{2}$ & $<2.0$ \\
\hline \hline R10789 glass beads & & $(8.1 \pm 0.1) \cdot 10^{2}$ & $(3.3 \pm 0.6) \cdot 10^{2}$ & $<4.1 \cdot 10^{2}$ & $(1.4 \pm 0.1) \cdot 10^{2}$ & $(1.4 \pm 0.1) \cdot 10^{3}$ & $<2.3$ \\
\hline
\end{tabular}

\subsubsection{The potassium compound used for photocathode production}

Most of the ${ }^{40} \mathrm{~K}$ in an assembled R10789 is presumed to come from the photocathode material, which is deposited on the back of the entrance window during the assembly process [11]. Chemicals used to make the photocathode material contain potassium, which contains the radioactive isotope ${ }^{40} \mathrm{~K}$ with a natural abundance of $117 \mathrm{ppm}$. Since potassium is an essential ingredient for the photocathode, ${ }^{39} \mathrm{~K}$-enriched potassium chromate from ATOX Co., Ltd. was used to produce the 
photocathode for the new R13111 PMT. Table 3 shows the RIs of two samples of potassium chromate, one without enrichment and the other enriched in ${ }^{39} \mathrm{~K}$. For the one without enrichment, the amount of ${ }^{40} \mathrm{~K}$ was $10 \pm 1 \mathrm{~Bq} / \mathrm{g}$. This corresponds to $99.8 \pm 0.7 \mathrm{ppm}$, which is consistent with the natural abundance within the systematic error of the HPGe measurements. In contrast ${ }^{39} \mathrm{~K}$-enriched potassium chromate has $84 \pm 4 \mathrm{mBq} / \mathrm{g}$, or $0.81 \pm 0.04 \mathrm{ppm}$ of ${ }^{40} \mathrm{~K}$, a reduction of more than two orders of magnitude.

Table 3. Results of HPGe measurements for $20 \mathrm{~g}$ of the photocathode material. The units are $\mu \mathrm{Bq} / \mathrm{g}$. Errors in the table include only statistical error. See the text for the systematic error.

\begin{tabular}{|c|r|r|r|r|r|r|}
\hline Samples & ${ }^{226} \mathrm{Ra}$ & $\begin{array}{r}{ }^{238} \mathrm{U} \\
\left({ }^{234} \mathrm{Th}\right)\end{array}$ & ${ }^{210} \mathrm{~Pb}$ & ${ }^{228} \mathrm{Ra}$ & ${ }^{40} \mathrm{~K}$ & ${ }^{60} \mathrm{Co}$ \\
\hline without enrichment & $<82$ & $<3.0 \cdot 10^{4}$ & $<8.8 \cdot 10^{4}$ & $<3.4 \cdot 10^{2}$ & $(1.0 \pm 0.1) \cdot 10^{7}$ & $<9.9 \cdot 10^{2}$ \\
\hline with enrichment & $<2.2 \cdot 10^{2}$ & $<1.3 \cdot 10^{3}$ & $<8.9 \cdot 10^{3}$ & $<1.9 \cdot 10^{2}$ & $(8.4 \pm 0.4) \cdot 10^{4}$ & $<52$ \\
\hline
\end{tabular}

\subsubsection{The aluminum seal}

Aluminum is used for the seal between the quartz window and the metal body of both the R10789 and the R13111. For the R10789, 98.5\% purity aluminum was used for the seal. During the XMASS $800 \mathrm{~kg}$ commissioning phase, we found that a large amount of the surface BG was caused by radioisotopes included in this aluminum seal. The relevant isotopes came from the top part of the ${ }^{238} \mathrm{U}$ decay chain and ${ }^{210} \mathrm{~Pb}$ [1]. For the R13111, we decided to use higher-purity, 5N aluminum (Nippon Light Metal Company, LTD.) for the seal. This reduced these isotopes substantially as shown in Table 4. The amount of ${ }^{238} \mathrm{U}$ measured by mass spectrometry shows three orders of magnitude reduction. For ${ }^{210} \mathrm{~Pb}$, more than about two orders of magnitude reduction was achieved. Other RIs are also reduced considerably by the increased purity of the new material. 
Table 4. Results of HPGe and mass spectrometry measurements for the aluminum seal materials. The units are $\mu \mathrm{Bq} / \mathrm{g}$. Errors for HPGe measurements in the table include only statistical error. See the text for the systematic error.

\begin{tabular}{|c|c|c|c|c|c|c|c|c|c|}
\hline Samples & ${ }^{226} \mathrm{Ra}$ & ${ }^{238} \mathrm{U}$ & ${ }^{210} \mathrm{~Pb}$ & ${ }^{228} \mathrm{Ra}$ & ${ }^{40} \mathrm{~K}$ & ${ }^{60} \mathrm{Co}$ & ${ }^{238} \mathrm{U}$ & ${ }^{232} \mathrm{Th}$ & $\begin{array}{r}\text { Method for } \\
{ }^{238} \mathrm{U} \text { and }{ }^{232} \mathrm{Th}\end{array}$ \\
\hline used in 2015 Production (5N purity) & $<10$ & $<51$ & $<1.0 \cdot 10^{2}$ & $13 \pm 5$ & $<1.8 \cdot 10^{2}$ & $<5.8$ & 2.5 & $4.1 \cdot 10^{-1}$ & GD-MS \\
\hline used in 2016 Production (5N purity) & $<2.8$ & $<37$ & $<66$ & $<2.5$ & $<34$ & $<9.6 \cdot 10^{-1}$ & 2.5 & $4.1 \cdot 10^{-1}$ & GD-MS \\
\hline used in R10789 PMT (98.5\% purity) & $<36$ & $(3.3 \pm 0.4) \cdot 10^{3}$ & $(7.7 \pm 6.0) \cdot 10^{3}$ & $(1.0 \pm 0.3) \cdot 10^{2}$ & $<7.1 \cdot 10^{2}$ & $<18$ & $3.9 \cdot 10^{3}$ & 74 & ICP-MS \\
\hline
\end{tabular}




\subsection{Resulting improvements}

\subsubsection{RIs of the PMT components}

We report the RIs in each component of the newly developed R13111 in this section. Table 1 shows a list of components measured by the HPGe detectors, together with the weight of each part (measured during the year 2015). We prepared large samples which were 30-500 times larger than the amount of material used for a single PMT in order to have the required sensitivity.

The amounts of ${ }^{226} \mathrm{Ra},{ }^{238} \mathrm{U}$ and ${ }^{210} \mathrm{~Pb}$ from the ${ }^{238} \mathrm{U}$ decay chain, ${ }^{228} \mathrm{Ra}$ from the ${ }^{232} \mathrm{Th}$ chain, and ${ }^{40} \mathrm{~K}$ and ${ }^{60} \mathrm{Co}$ were determined using the HPGe detectors. Mass-spectrometry was used to measure the amounts of ${ }^{238} \mathrm{U},{ }^{232} \mathrm{Th}$, and ${ }^{40} \mathrm{~K}$ assuming the natural abundance of isotopes for each element. These mass-spectrometry measurements were performed to increase the sensitivity for materials with large volumes per PMT. Mass spectrometers have better sensitivity than HPGe, which becomes relevant when large total amounts of material increase the impact of even tiny contaminations.

The results of the measured RIs for PMTs assembled in years 2015 and 2016 are shown separately in Table 5 and Table 6, respectively. In case the center value does not exceed twice the statistical error, or is negative, we regarded the result as consistent with zero and set an upper limit calculated as $\max \{0$, the central value $\}+1.28 \times$ statistical error. Though the PMTs produced in both years used the same materials from the same companies, the observed numbers are different for many parts. This is because the production lots of those parts are not the same. For ${ }^{226} \mathrm{Ra}$, ${ }^{210} \mathrm{~Pb},{ }^{228} \mathrm{Ra}$, and ${ }^{60} \mathrm{Co}$, the total activities of the PMT components are also shown in Tables 5 and 6. In calculating of the total radioactivity, we summed up the central values of each component, with the sign of each value included in the sum. To calculate the total activity of ${ }^{238} \mathrm{U}$ and ${ }^{40} \mathrm{~K}$, we used the mass spectrometer results instead of HPGe results, if the mass spectrometer results exist and the sensitivity of the results were better than that of the HPGe result. These are shown in Table 7 as Part-by-part sum (2015) and Part-by-part sum (2016). Since the errors in the table show only statistical error, systematic errors would add another $+30 \%$ to $-10 \%$ to the HPGe, $\pm 30 \%$ to the GD-MS, and $\pm 10 \%$ to the ICP-MS measurements results.

Among the components in Tables 5 and 6, some stainless-steel parts in particular bring large contributions of ${ }^{226} \mathrm{Ra},{ }^{228} \mathrm{Ra}$ and ${ }^{60} \mathrm{Co}$ compared to the total. Most prominent are the cathode, the disk, the 1st dynode, the 2nd-11th dynodes, and the shield. Such stainless-steel parts also differ noticeably in their respective 2015 and 2016 RI contributions. To manufacture them, we used commercial products that have large variations in the RIs. This also applies for the cobalt-free metal used in the PMT body and the stem during 2015, which have large values of ${ }^{228} \mathrm{Ra}$ and ${ }^{60} \mathrm{Co}$, while the same material used in 2016 shows smaller amounts. The glass parts (the glass beads and the disk feedthrough) still have large amounts of ${ }^{226} \mathrm{Ra},{ }^{228} \mathrm{Ra}$ and especially ${ }^{40} \mathrm{~K}$, though we largely reduced the RIs in the glass beads. 
Table 5. Result of the measurement for each part used for R13111 production in 2015. The units are $\mu \mathrm{Bq} / \mathrm{PMT}$. Errors in the table include only statistical error. See the text for the systematic error.

\begin{tabular}{|c|c|c|c|c|c|c|c|c|c|c|}
\hline \multirow[b]{2}{*}{ Samples } & \multicolumn{6}{|c|}{ results of HPGe } & \multicolumn{4}{|c|}{ result of the mass spectrometry } \\
\hline & $226 \mathrm{Ra}$ & $238 \mathrm{U}$ & $210 \mathrm{~Pb}$ & $228 \mathrm{Ra}$ & ${ }^{40} \mathrm{~K}$ & ${ }^{60} \mathrm{Co}$ & ${ }^{238} \mathrm{U}$ & ${ }^{232} \mathrm{Th}$ & ${ }^{40} \mathrm{~K}$ & $\begin{array}{l}\text { Method for } \\
{ }^{238} \mathrm{U} \text { and }{ }^{232} \mathrm{Th}\end{array}$ \\
\hline Body & $<1.0 \cdot 10^{2}$ & $<3.1 \cdot 10^{3}$ & $<1.2 \cdot 10^{5}$ & $(1.2 \pm 0.4) \cdot 10^{2}$ & $<5.5 \cdot 10^{2}$ & $(1.5 \pm 0.2) \cdot 10^{2}$ & $2.1 \cdot 10^{2}$ & $<17$ & - & GD-MS \\
\hline Stem & $<1.0 \cdot 10^{2}$ & $<3.0 \cdot 10^{3}$ & $<8.8 \cdot 10^{4}$ & $<1.4 \cdot 10^{2}$ & $<8.4 \cdot 10^{2}$ & $64 \pm 18$ & $5.5 \cdot 10^{2}$ & $<26$ & $<2.0$ & GD-MS \\
\hline Stem pin & $<24$ & $<2.8 \cdot 10^{2}$ & $<9.2 \cdot 10^{3}$ & $<27$ & $<2.2 \cdot 10^{2}$ & $86 \pm 9$ & $<2.0$ & $<6.5 \cdot 10^{-1}$ & $<2.5 \cdot 10^{-1}$ & GD-MS \\
\hline Glass beads & $<34$ & $<1.7 \cdot 10^{2}$ & $<4.5 \cdot 10^{2}$ & $<22$ & $(3.9 \pm 1.6) \cdot 10^{2}$ & $<4.7$ & - & - & - & - \\
\hline Quartz Window & $<2.0 \cdot 10^{2}$ & $<1.6 \cdot 10^{3}$ & $<6.8 \cdot 10^{3}$ & $<1.7 \cdot 10^{2}$ & $<2.8 \cdot 10^{3}$ & $<49$ & $<20$ & $<6.7$ & $7.6 \cdot 10^{-2}$ & ICP-MS \\
\hline Quartz dynode support & $<44$ & $<7.9 \cdot 10^{2}$ & $<3.5 \cdot 10^{4}$ & $<32$ & $<5.3 \cdot 10^{2}$ & $<8.1$ & $<3.0$ & $<9.8 \cdot 10^{-1}$ & 7.8 & ICP-MS \\
\hline Quartz spacer & $<20$ & $<1.4 \cdot 10^{2}$ & $<7.0 \cdot 10^{2}$ & $<20$ & $<1.4 \cdot 10^{2}$ & $<4.3$ & $<1.7 \cdot 10^{-1}$ & $<5.7 \cdot 10^{-2}$ & $4.6 \cdot 10^{-1}$ & ICP-MS \\
\hline Al seal & $<9.0$ & $<46$ & $<94$ & $11 \pm 5$ & $<1.7 \cdot 10^{2}$ & $<5.2$ & 2.2 & $3.7 \cdot 10^{-1}$ & - & GD-MS \\
\hline Kovar ring & $<29$ & $<7.2 \cdot 10^{2}$ & $<2.1 \cdot 10^{4}$ & $<28$ & $<2.3 \cdot 10^{2}$ & $37 \pm 8$ & $<4.6$ & $<1.5$ & $<5.7 \cdot 10^{-1}$ & GD-MS \\
\hline Sb for evaporation & $15 \pm 6$ & $<40$ & $<94$ & $<6.1$ & $<1.4 \cdot 10^{2}$ & $<2.2$ & - & - & - & - \\
\hline $\mathrm{Al}$ for evaporation & $<4.2$ & $<21$ & $<33$ & $<5.8$ & $<83$ & $<1.1$ & $1.6 \cdot 10^{-1}$ & $2.6 \cdot 10^{-2}$ & - & GD-MS \\
\hline Disk feedthrough & $11 \pm 2$ & $<45$ & $<4.8 \cdot 10^{2}$ & $(5.5 \pm 2.4)$ & $71 \pm 34$ & $10 \pm 1$ & - & - & - & - \\
\hline Lead wire & $42 \pm 8$ & $<2.9 \cdot 10^{2}$ & $<6.7 \cdot 10^{3}$ & $<21$ & $<1.1 \cdot 10^{2}$ & $<4.0$ & - & - & - & - \\
\hline Screw, nut, washer, getter stopper & $<12$ & $<2.8 \cdot 10^{2}$ & $<3.6 \cdot 10^{3}$ & $<25$ & $<1.3 \cdot 10^{2}$ & $<3.9$ & $1.3 \cdot 10^{2}$ & 2.8 & 1.1 & GD-MS \\
\hline Cathode & $(1.3 \pm 0.3) \cdot 10^{2}$ & $<8.2 \cdot 10^{2}$ & $<5.8 \cdot 10^{3}$ & $67 \pm 24$ & $<4.6 \cdot 10^{2}$ & $23 \pm 10$ & 23 & 11 & 1.7 & GD-MS \\
\hline Dynode 1st stage & $(1.1 \pm 0.3) \cdot 10^{2}$ & $<1.1 \cdot 10^{3}$ & $<4.7 \cdot 10^{3}$ & $<66$ & $<3.8 \cdot 10^{2}$ & $<30$ & $1.0 \cdot 10^{2}$ & 17 & $<6.6 \cdot 10^{-1}$ & GD-MS \\
\hline Shield & $<52$ & $<9.2 \cdot 10^{2}$ & $<8.5 \cdot 10^{3}$ & $<55$ & $<5.3 \cdot 10^{2}$ & $<20$ & $1.6 \cdot 10^{2}$ & 27 & $<1.0$ & GD-MS \\
\hline Spring, Number plate & $<8.8$ & $<1.2 \cdot 10^{2}$ & $<1.9 \cdot 10^{3}$ & $<9.1$ & $<1.2 \cdot 10^{2}$ & $<3.5$ & 1.8 & $9.4 \cdot 10^{-1}$ & $<4.4 \cdot 10^{-1}$ & GD-MS \\
\hline Anode mesh & $<16$ & $<1.4 \cdot 10^{2}$ & $<4.7 \cdot 10^{3}$ & $<11$ & $<2.3 \cdot 10^{2}$ & $<3.3$ & - & - & - & - \\
\hline Disk Stopper & $<12$ & $<6.7 \cdot 10^{2}$ & $<1.5 \cdot 10^{4}$ & $<14$ & $<1.7 \cdot 10^{2}$ & $<4.5$ & - & - & - & - \\
\hline Dynode 1st stage mesh & $<22$ & $<89$ & $<2.7 \cdot 10^{2}$ & $<19$ & $<2.4 \cdot 10^{2}$ & $<5.1$ & - & - & - & - \\
\hline Disk & $95 \pm 36$ & $<9.5 \cdot 10^{2}$ & $<1.5 \cdot 10^{4}$ & $94 \pm 35$ & $<4.9 \cdot 10^{2}$ & $94 \pm 17$ & $1.1 \cdot 10^{2}$ & 53 & 8.1 & GD-MS \\
\hline Getter & $<5.3$ & $<81$ & $<6.1 \cdot 10^{2}$ & $<5.2$ & $<1.2 \cdot 10^{2}$ & $<1.9$ & - & - & - & - \\
\hline Exhaust tube & $<33$ & $<1.4 \cdot 10^{2}$ & $<1.1 \cdot 10^{3}$ & $<23$ & $<4.5 \cdot 10^{2}$ & $<4.6$ & - & - & - & - \\
\hline Sum & $(6.2 \pm 1.7) \cdot 10^{2}$ & - & $<1.6 \cdot 10^{5}$ & $(4.8 \pm 1.5) \cdot 10^{2}$ & - & $(4.8 \pm 0.6) \cdot 10^{2}$ & - & - & - & - \\
\hline
\end{tabular}


Table 6. Result of the measurement for each part used for R13111 production in 2016. The units are $\mu \mathrm{Bq} / \mathrm{PMT}$. Errors in the table include only statistical error. See the text for the systematic error.

\begin{tabular}{|c|c|c|c|c|c|c|c|c|c|c|}
\hline \multirow[b]{2}{*}{ Samples } & \multicolumn{6}{|c|}{ results of HPGe } & \multicolumn{4}{|c|}{ result of the mass spectrometry } \\
\hline & ${ }^{226} \mathrm{Ra}$ & ${ }^{238} \mathrm{U}$ & ${ }^{210} \mathrm{~Pb}$ & ${ }^{228} \mathrm{Ra}$ & ${ }^{40} \mathrm{~K}$ & ${ }^{60} \mathrm{Co}$ & ${ }^{238} \mathrm{U}$ & ${ }^{232} \mathrm{Th}$ & ${ }^{40} \mathrm{~K}$ & $\begin{array}{r}\text { Method for } \\
{ }^{238} \mathrm{U} \text { and }{ }^{232} \mathrm{Th}\end{array}$ \\
\hline Body & $<55$ & $<2.3 \cdot 10^{3}$ & $<7.3 \cdot 10^{4}$ & $<1.2 \cdot 10^{2}$ & $<5.1 \cdot 10^{2}$ & $<36$ & $<73$ & $<17$ & 55 & GD-MS \\
\hline Stem & $<1.1 \cdot 10^{2}$ & $<4.1 \cdot 10^{3}$ & $<7.4 \cdot 10^{4}$ & $<1.0 \cdot 10^{2}$ & $<4.8 \cdot 10^{2}$ & $<33$ & $5.5 \cdot 10^{2}$ & $<26$ & $<2.0$ & GD-MS \\
\hline Stem pin & $<7.1$ & $<2.7 \cdot 10^{2}$ & $<1.7 \cdot 10^{3}$ & $<9.2$ & $<86$ & $84 \pm 5$ & $<1.8$ & $<6.0 \cdot 10^{-1}$ & $<2.3 \cdot 10^{-1}$ & GD-MS \\
\hline Glass beads & $16 \pm 3$ & $<1.5 \cdot 10^{2}$ & $<5.9 \cdot 10^{2}$ & $9.8 \pm 3.4$ & $(6.7 \pm 0.6) \cdot 10^{2}$ & $<1.2$ & $<1.2 \cdot 10^{2}$ & $<41$ & $<6.8 \cdot 10^{2}$ & ICP-MS \\
\hline Quartz window & $<87$ & $<1.1 \cdot 10^{3}$ & $<2.5 \cdot 10^{4}$ & $<54$ & $<2.9 \cdot 10^{2}$ & $<28$ & $<20$ & $<6.7$ & $7.6 \cdot 10^{-2}$ & ICP-MS \\
\hline Quartz dynode support & $<31$ & $<2.5 \cdot 10^{2}$ & $<2.8 \cdot 10^{3}$ & $<16$ & $<1.2 \cdot 10^{2}$ & $<8.4$ & $<3.0$ & $<9.9 \cdot 10^{-1}$ & 7.9 & ICP-MS \\
\hline Quartz spacer & $<20$ & $<1.4 \cdot 10^{2}$ & $<7.0 \cdot 10^{2}$ & $<20$ & $<1.4 \cdot 10^{2}$ & $<4.3$ & $<1.7 \cdot 10^{-1}$ & $<5.7 \cdot 10^{-2}$ & $4.6 \cdot 10^{-1}$ & ICP-MS \\
\hline $\mathrm{Al}$ seal & $<2.5$ & $<33$ & $<60$ & $<2.2$ & $<31$ & $<8.7 \cdot 10^{-1}$ & 2.2 & $3.7 \cdot 10^{-1}$ & - & GD-MS \\
\hline Kovar ring & $21 \pm 6$ & $<1.3 \cdot 10^{2}$ & $<2.6 \cdot 10^{4}$ & $<9.4$ & $<97$ & $19 \pm 3$ & $<2.2 \cdot 10^{2}$ & $<72$ & 5.5 & GD-MS \\
\hline Sb for evaporation & $<10$ & $<60$ & $<2.2 \cdot 10^{2}$ & $<6.9$ & $<1.0 \cdot 10^{2}$ & $<1.2$ & - & - & - & - \\
\hline $\mathrm{Al}$ for evaporation & $<4.2$ & $<21$ & $<33$ & $<5.8$ & $<83$ & $<1.1$ & $1.6 \cdot 10^{-1}$ & $2.6 \cdot 10^{-2}$ & - & GD-MS \\
\hline Disk feedthrough & $11 \pm 2$ & $<45$ & $<4.8 \cdot 10^{2}$ & $5.5 \pm 2.4$ & $71 \pm 34$ & $10 \pm 1$ & - & - & - & - \\
\hline Lead wire, ribbons, getter stopper & $<25$ & $<2.5 \cdot 10^{2}$ & $<1.4 \cdot 10^{3}$ & $<14$ & $<4.6 \cdot 10^{2}$ & $<6.5$ & 54 & 1.7 & $9.2 \cdot 10^{-1}$ & GD-MS \\
\hline Screw, nut, washer & $<18$ & $<74$ & $<3.3 \cdot 10^{2}$ & $<6.1$ & $<89$ & $<4.6$ & 40 & 15 & 2.1 & GD-MS \\
\hline Cathode & $5.4 \pm 2.5$ & $<2.4 \cdot 10^{2}$ & $<4.8 \cdot 10^{3}$ & $<6.2$ & $<80$ & $(1.9 \pm 0.9)$ & $<3.0$ & 4.9 & $<3.7 \cdot 10^{-1}$ & GD-MS \\
\hline Dynode 1st stage & $<33$ & $<2.5 \cdot 10^{2}$ & $<8.8 \cdot 10^{2}$ & $<20$ & $<3.1 \cdot 10^{2}$ & $<14$ & $<2.8 \cdot 10^{2}$ & $<93$ & $<14$ & GD-MS \\
\hline Dynode (2- 11 stage) & $(1.0 \pm 0.4) \cdot 10^{2}$ & $<5.5 \cdot 10^{2}$ & $<2.5 \cdot 10^{3}$ & $<51$ & $<5.6 \cdot 10^{2}$ & $32 \pm 13$ & $<9.0$ & $<3.0$ & $\begin{array}{l}<1.1 \\
\end{array}$ & GD-MS \\
\hline Dynode (12th stage) & $<20$ & $<1.0 \cdot 10^{2}$ & $<2.4 \cdot 10^{2}$ & $<13$ & $<1.3 \cdot 10^{2}$ & $<2.7$ & 2.1 & $9.0 \cdot 10^{-1}$ & $8.6 \cdot 10^{-1}$ & GD-MS \\
\hline Shield & $(1.0 \pm 0.2) \cdot 10^{2}$ & $<7.6 \cdot 10^{2}$ & $<7.7 \cdot 10^{3}$ & $<26$ & $<3.0 \cdot 10^{2}$ & $<12$ & $<4.1 \cdot 10^{2}$ & $<1.4 \cdot 10^{2}$ & $<21$ & GD-MS \\
\hline Spring, number plate & $<8.8$ & $<1.2 \cdot 10^{2}$ & $<1.9 \cdot 10^{3}$ & $<9.1$ & $<1.2 \cdot 10^{2}$ & $<3.5$ & 1.8 & $9.4 \cdot 10^{-1}$ & $4.4 \cdot 10^{-1}$ & GD-MS \\
\hline Anode mesh, Disk Stopper, Dynode 1st stage mesh & $23 \pm 9$ & $<2.1 \cdot 10^{2}$ & $<5.3 \cdot 10^{3}$ & $<20$ & $<2.6 \cdot 10^{2}$ & $<3.8$ & - & - & - & - \\
\hline Disk & $<20$ & $<1.0 \cdot 10^{3}$ & $<4.2 \cdot 10^{3}$ & $<17$ & $<4.1 \cdot 10^{2}$ & $11 \pm 5$ & $<16$ & $<5.1$ & $<2.0$ & GD-MS \\
\hline Getter & $<5.3$ & $<81$ & $<6.1 \cdot 10^{2}$ & $<5.2$ & $<1.2 \cdot 10^{2}$ & $<1.9$ & - & - & - & - \\
\hline Exhaust tube & $<11$ & $<2.6 \cdot 10^{2}$ & $<7.2 \cdot 10^{3}$ & $<14$ & $<2.4 \cdot 10^{2}$ & $<4.6$ & - & - & - & - \\
\hline Sum & $(3.9 \pm 1.0) \cdot 10^{2}$ & - & $<1.1 \cdot 10^{5}$ & $<2.0 \cdot 10^{2}$ & - & $(1.9 \pm 0.4) \cdot 10^{2}$ & - & - & - & - \\
\hline
\end{tabular}




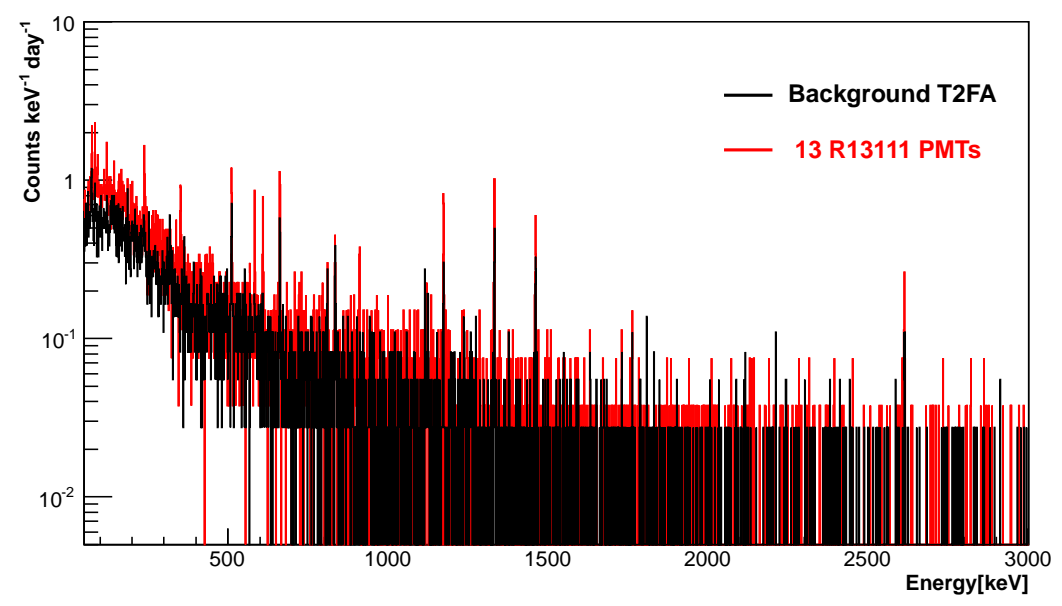

Figure 4. The energy spectra for 13 R13111 PMTs measured over 27 days (red) with the 36 day BG spectra (black). For the PMTs produced in 2015 with the ${ }^{39} \mathrm{~K}$-enriched photocathode material. The bin width is 1 $\mathrm{keV}$.

\subsubsection{RIs of fully assembled PMTs}

We also carried out the RI measurement of PMTs after production to also evaluate possible contamination during the assembling process and asses the overall reduction of ${ }^{40} \mathrm{~K}$ in the photocathode; the ${ }^{39} \mathrm{~K}$-enriched potassium compound is used only in the photocathode and the RIs of the finished photocathode cannot be measured before assembling the PMT. The effect of the ${ }^{39} \mathrm{~K}$ enrichment was examined by measuring two variants of the R13111 PMT: one with natural potassium and the other with ${ }^{39} \mathrm{~K}$-enriched potassium.

Figure 4 shows the energy spectrum of 13 such PMTs of the 2015 variant with the ${ }^{39} \mathrm{~K}$-enriched photocathode. Table 7 shows the results for the produced new PMT varieties. In particular, it shows RIs for 2015 with the natural potassium, 2015 with ${ }^{39} \mathrm{~K}$-enriched potassium, and 2016 with ${ }^{39} \mathrm{~K}$-enriched potassium. The sum of the radioactivity from component values in Table 7 is taken from Tables 5 and 6 . To calculate the ${ }^{238} \mathrm{U}$ sum and ${ }^{40} \mathrm{~K}$ sum, we use the mass spectrometry results if this results exist and the sensitivity of results is better than that of HPGe results.

The results for the PMTs assembled in 2015 with the enriched potassium and those assembled in 2016 with the enriched potassium are consistent with each other. Differences are less than two sigma. However, for each year, the measurements of RIs for parts often differ from other years due to the different production lots they were drawn from (see section 3.3.1). Based on our screening, we controlled the total RI differences which came from using different lot parts to the level of our measurements.

As summarized in Table 7, we achieved a large reduction of ${ }^{40} \mathrm{~K}$ contamination by using ${ }^{39} \mathrm{~K}$ enriched potassium for the photocathode. PMTs produced early in 2015 were made with potassium of natural isotope abundance, leading to $18.5 \pm 1.4 \mathrm{mBq} / \mathrm{PMT}$ from ${ }^{40} \mathrm{~K}$. Later PMT batches produced with ${ }^{39} \mathrm{~K}$ enriched potassium yielded an upper limit of $1.4 \mathrm{mBq} / \mathrm{PMT}$ for PMTs produced in 2015 and a measurement of $2.0 \pm 0.5 \mathrm{mBq} / \mathrm{PMT}$ for those produced in 2016 . Using ${ }^{39} \mathrm{~K}$ enriched potassium thus reduced ${ }^{40} \mathrm{~K}$ decays in these PMTs by almost one order of magnitude. 
Table 7. PMT measurement results. The units are mBq/PMT. Errors in the table include only statistical error. See the text for the systematic error

\begin{tabular}{|c|r|r|r|r|r|r|}
\hline mBq/PMT & ${ }^{226} \mathrm{Ra}$ & ${ }^{238} \mathrm{U}$ & ${ }^{210} \mathrm{~Pb}$ & ${ }^{228} \mathrm{Ra}$ & ${ }^{40} \mathrm{~K}$ & ${ }^{60} \mathrm{Co}$ \\
\hline \hline $\begin{array}{c}\text { Assembled PMT (2015) } \\
\text { with natural K }\end{array}$ & $(4.4 \pm 0.9) \cdot 10^{-1}$ & $<2.7$ & $<56$ & $(3.5 \pm 0.8) \cdot 10^{-1}$ & $18.5 \pm 1.4$ & $(2.3 \pm 0.6) \cdot 10^{-1}$ \\
\hline $\begin{array}{c}\text { Assembled PMT (2015) } \\
\text { with }{ }^{39} \text { K-enriched }\end{array}$ & $(3.8 \pm 0.7) \cdot 10^{-1}$ & $<1.6$ & $<32$ & $(2.9 \pm 0.6) \cdot 10^{-1}$ & $<1.4$ & $(2.2 \pm 0.5) \cdot 10^{-1}$ \\
\hline Part-by-part sum (2015) & $(6.2 \pm 1.7) \cdot 10^{-1}$ & $<2.2$ & $<1.6 \cdot 10^{2}$ & $(4.8 \pm 1.5) \cdot 10^{-1}$ & $<1.3$ & $(4.8 \pm 0.6) \cdot 10^{-1}$ \\
\hline \hline $\begin{array}{c}\text { Assembled PMT (2016) } \\
\text { with }{ }^{39} \text { K-enriched }\end{array}$ & $(4.4 \pm 0.6) \cdot 10^{-1}$ & $<1.4$ & $<24$ & $(2.0 \pm 0.6) \cdot 10^{-1}$ & $2.0 \pm 0.5$ & $(1.3 \pm 0.4) \cdot 10^{-1}$ \\
\hline \hline Part-by-part sum (2016) & $(3.9 \pm 1.0) \cdot 10^{-1}$ & $<2.0$ & $<1.1 \cdot 10^{2}$ & $<2.0 \cdot 10^{-1}$ & $<1.0$ & $(1.9 \pm 0.4) \cdot 10^{-1}$ \\
\hline
\end{tabular}

To study the contamination during the production process, we compare the sum of all parts to the measurements of assembled PMTs. Table 7 shows the sum of all parts and the assembled PMTs for the year 2015 and 2016 with the enriched potassium. Among these numbers only ${ }^{60} \mathrm{Co}$ for the sum of all parts for 2015 seems to hint at some inconsistency: $0.48 \pm 0.06 \mathrm{mBq} / \mathrm{PMT}$ for ${ }^{60} \mathrm{Co}$ is significantly larger than the measurements on the assembled PMTs. This difference is explained by the decay of ${ }^{60} \mathrm{Co}$. The components were measured in 2015 , while the assembled PMT measurements were made in 2018, after the installation of the ultra-low-BG HPGe detector. After assembly, the PMTs were stored in the underground where the flux of cosmic-ray muons is much smaller and ${ }^{60} \mathrm{Co}$ cosmogenic production is negligible compared to that at ground level. Three years decrease the amount of ${ }^{60} \mathrm{Co}$ to $33 \%$, and thus the corrected amount of ${ }^{60} \mathrm{Co}$ for the sum of components in 2018 then becomes $0.32 \pm 0.04 \mathrm{mBq} / \mathrm{PMT}$, which is consistent with the measurements of PMTs assembled in 2018. Since no significant difference between the sum of the components and the assembled PMTs was identified for all RI components, we concluded that there was no significant contamination introduced during the assembling process.

\subsubsection{Comparison to other PMT used for LXe detectors}

Table 8 shows the photocathode area normalized RI summaries for the R13111 PMT and the R10789 PMT. The values for the R10789 PMT are normalized using its photocathode area of $24.6 \mathrm{~cm}^{2}$. The R13111 PMT values were normalized with the projected area its photocathode presents for normal incidence at its center: a circle of $70 \mathrm{~mm}$ diameter with an area of $38.5 \mathrm{~cm}^{2}$. When compared to the R10789 PMT, ${ }^{226} \mathrm{Ra}$, and ${ }^{228} \mathrm{Ra}$ were reduce by about a factor of 5 , the ${ }^{40} \mathrm{~K}$ by a factor larger than 7 and ${ }^{60} \mathrm{Co}$ by a factor larger than 20. Thus with the R13111 PMT we did indeed achieve a large reduction of RI.

Table 9 compares the RIs between the R13111 PMT variants presented in this work and the R11410 PMT used by XENON1T[15], PandaX[3] and LUX[19]. Almost all PMT RIs are lowest for our R13111 among these PMTs. Only ${ }^{226}$ Ra for the 2016 R13111 PMTs is larger than the R11410-10 (LUX) number, though the levels are quite similar. 
Table 8. Results of measurements for the R13111 PMT and the R10789 PMT in $\mu \mathrm{Bq} / \mathrm{cm}^{2}$. The numbers are normalized by photocathode area. Errors in the table are statistical only. See the text for the systematic error.

\begin{tabular}{|c|r|r|r|r|}
\hline$\mu \mathrm{Bq} / \mathrm{cm}^{2}$ & ${ }^{226} \mathrm{Ra}$ & ${ }^{228} \mathrm{Ra}$ & ${ }^{40} \mathrm{~K}$ & ${ }^{60} \mathrm{Co}$ \\
\hline \hline Assembled PMT (2015) & $9.9 \pm 1.8$ & $7.5 \pm 1.6$ & $<36$ & $5.7 \pm 1.3$ \\
Assembled PMT (2016) & $11 \pm 2$ & $5.2 \pm 1.6$ & $52 \pm 13$ & $3.4 \pm 1.0$ \\
\hline $\mathrm{R} 10789$ & $49 \pm 12$ & $<32$ & $(3.7 \pm 0.9) \cdot 10^{2}$ & $(1.1 \pm 0.1) \cdot 10^{2}$ \\
\hline
\end{tabular}

Table 9. RI comparison between the R13111 PMT and other PMTs used by other groups. The units are $\mu \mathrm{Bq} / \mathrm{PMT}$.

\begin{tabular}{|l|r|r|r|r|r|}
\hline$\mu$ Bq/PMT & ${ }^{226} \mathrm{Ra}$ & ${ }^{238} \mathrm{U}$ & ${ }^{228} \mathrm{Ra}$ & ${ }^{40} \mathrm{~K}$ & ${ }^{60} \mathrm{Co}$ \\
\hline \hline R13111 in 2015 & $(3.8 \pm 0.7) \cdot 10^{2}$ & $<1.6 \cdot 10^{3}$ & $(2.9 \pm 0.6) \cdot 10^{2}$ & $<1.4 \cdot 10^{3}$ & $(2.2 \pm 0.5) \cdot 10^{2}$ \\
R13111 in 2016 & $(4.4 \pm 0.6) \cdot 10^{2}$ & $<1.4 \cdot 10^{3}$ & $(2.0 \pm 0.6) \cdot 10^{2}$ & $(2.0 \pm 0.5) \cdot 10^{3}$ & $(1.3 \pm 0.4) \cdot 10^{2}$ \\
\hline R11410-21(XENON1T)[15] & $(5.2 \pm 1.0) \cdot 10^{2}$ & $<1.3 \cdot 10^{4}$ & $(3.9 \pm 1.0) \cdot 10^{2}$ & $(1.2 \pm 0.2) \cdot 10^{4}$ & $(7.4 \pm 1.0) \cdot 10^{2}$ \\
R11410-10(PandaX)[3] & $<7.2 \cdot 10^{2}$ & - & $<8.3 \cdot 10^{2}$ & $(1.5 \pm 0.8) \cdot 10^{4}$ & $(3.4 \pm 0.4) \cdot 10^{3}$ \\
R11410-10(LUX)[19] & $<4.0 \cdot 10^{2}$ & $<6.0 \cdot 10^{3}$ & $<3.0 \cdot 10^{2}$ & $<8.3 \cdot 10^{3}$ & $(2.0 \pm 0.2) \cdot 10^{3}$ \\
\hline
\end{tabular}

\section{Afterpulse study related to the Xe leak problem}

For the R11410, which has many structures in common with the R13111, Xe afterpulses caused by Xe leaking into the PMTs were previously reported by XENON1T and PandaX [13, 14]. Such Xe leaks are a serious problem for LXe detectors, since afterpulses interfere with and confuse the dark matter or other physics signals; a large enough leak ultimately makes it impossible to operate such a PMT. Therefore, we examined the Xe leak for the R13111 PMT and observed a similar leak as reported for R11410. We carried out a detailed study of the Xe afterpulse rate to solve this problem.

\subsection{Identification of the leaking component}

We observed no Xe leak in 642 R10789 PMTs in XMASS-I, while leaks were observed in both the R13111 and the R11410 PMT. We compared the structure of these three PMTs and narrowed down the possible causes. First, we compared the R10789 with the R13111. The components modified from the R10789 to the R13111 could be the cause. Among the modifications, there were two candidates which might have caused the leak. One is the seal between the window and the body, the other are the glass beads at the stem. The R13111 PMT uses $5 \mathrm{~N}$ aluminum for the seal while the R10789 PMT uses $98.5 \%$ aluminum. The glass beads are the electrical insulation at the feedthroughs at the stem and changed to ones made from high-purity ingredients in the R13111 PMT. Next we compared the R13111 with the R11410. We hypothesized that the cause of the leak would be common in these two PMTs. From this second comparison, we narrowed down the possible causes to the $5 \mathrm{~N}$ aluminum seal which is used in both PMTs. The high purity glass beads are not used in the R11410 PMT. Hence, the surfaces of the seals in the assembled R10789 and the R13111 PMT were examined in detail and it was found that the seal surface of the R13111 PMT had many tiny scratches compared to that of the R10789 PMT. Consequently, we investigated the following two hypothetical causes of the leak; one is the usage of high-purity $5 \mathrm{~N}$ aluminum itself 

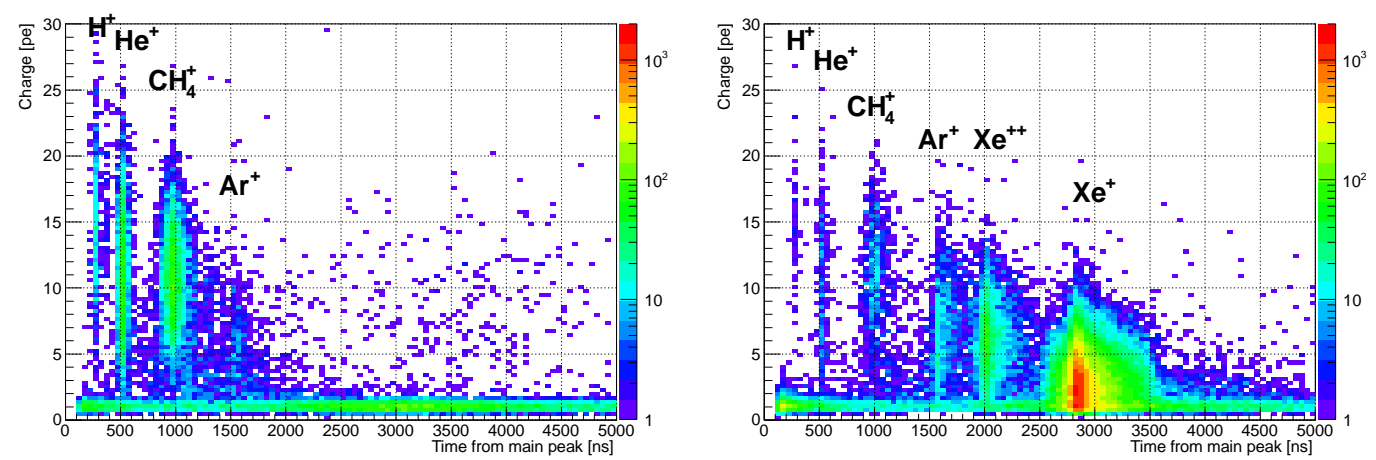

Figure 5. Afterpulse charge versus time delay from a light source signal for a normal PMT (left) and a PMT with Xe leak(right) at room temperature. Afterpulses from different ions of the residual gas in the PMTs vacuum were observed above the one photoelectron band at their respective expected timing.

and the other is a deterioration of the seal surface condition caused by the exchange itself not by the purity change.

To check this, we prepared two types of the R11410 PMT. One uses lower purity $4 \mathrm{~N}$ aluminum for the seal, the other uses the usual $5 \mathrm{~N}$ aluminum, however handling of the seal during the PMT assembling process was revised to pay special attention to the surface of the seal.

\subsection{Setup of the study}

To examine the afterpulse in a Xe atmosphere, we prepared a special chamber inside of which the PMTs could be cooled down to the LXe temperature of about -100 degree Celsius in a gaseous Xe atmosphere. By applying multiple temperature cycles of cooling and warming, we examined the afterpulses caused by ions of the residual gas. We applied at least three cooling cycles to each PMT. A light source (LED or laser diode) was adjusted to a level in which the PMT mostly observes just one photoelectron per flash of the respective light source. Afterpulses which follow the one photoelectron light source signal were recorded with FADCs and examined. Figure 5 shows the afterpulse charge against the time delay from the light source signal for a PMT without the Xe leak (left hand side) and one with a Xe leak (right hand side). All PMTs were operated at a gain of $1 \times$ $10^{7}$ in the study.

\subsection{Results of the study}

The number of PMTs that developed leaks after the temperature cycles in the study is summarized in Table 10. The Xe leaking PMTs were separated into two categories by their respective afterpulse rate, one is "large leak" and the other is "small leak". The afterpulse rate is the probability of afterpulse observation after one light source signal. We found that these two categories have different properties. For the "large leak" category the Xe afterpulse rate increased with every cooling cycle. The Xe afterpulse rate of all the large leak PMTs exceeded $0.1 \%$ after the third cooling cycle. For the "small leakâĂİ category, the Xe afterpulse rates are below $0.1 \%$ for all the small leak PMT even after the third cooling cycle. There was no increase in the afterpulse rate by the second and the third cooling cycle. We confirmed this property of the small leak category 
by applying additional cooling cycles to PMTs in this category. After applying seven additional cooling cycles to 10 small leak R13111 and one R11410 PMTs, we observed no change in their afterpulse rate.

Thirty of the 58 examined R13111 PMTs fabricated with 5N aluminum were also measured in liquid Xe rather than only cold gaseous Xe. Seven large and 3 small leaks among these 30 PMTs show no significant difference from 9 large and 7 small leaks observed for the other 28 PMTs tested only in gaseous Xe. The R11410 PMTs were only examined in gaseous Xe.

Out of 58 R13111 PMTs 16 developed large leaks and 10 small leaks during the cooling cycles. When compared to the numbers for R11410 with $5 \mathrm{~N}$ aluminum reported as 7 large leaks and one small leak among 44 PMTs [15], the fraction of Xe leaks for R13111 appears to be larger than that of R11410. The fraction of large leaks is $28 \pm 6 \%$ for R13111 and $16 \pm 6 \%$ for R11410, both with $5 \mathrm{~N}$ Al. For the $4 \mathrm{~N}$ aluminum R11410 PMT, we observed no large leak and one small leak. The probability of a large leak is less than $10 \%$ with $90 \% \mathrm{CL}$, assuming the observed fraction of the leak represents the probability of a leak. We also assumed no probability difference among PMT production batches if we keep the same assembling process. Though the significance is not large, the use of lower purity aluminum for the seal reduce the leak probability. The same improvement can be seen for the PMTs with $5 \mathrm{~N}$ aluminum that were prepared with special attention to the seal with an ever greater level of confidence. For these PMTs, neither large nor small leaks were observed in 28 PMTs. Consequently, the probability of large leak is less than $8 \%$.

We studied and confirmed more leaks in PMTs which used 5N aluminum for their seals than the ones which used $4 \mathrm{~N}$ aluminum. This suggests that the exchange of the sealing material to $5 \mathrm{~N}$ aluminum is a cause of the Xe leaks. We also observed that it is possible to suppress leakage when using $5 \mathrm{~N}$ aluminum by applying appropriate surface care. Since the sealing structure is common in the R11410 and the R13111, this result obtained with the R11410 PMT should also apply for the R13111 PMT.

Table 10. Results of the Xe leak test for the R13111 PMTs and the R11410 PMTs

\begin{tabular}{|c|l|r|r|r|r|}
\hline PMT & Sealing type & Tested number & $\begin{array}{r}\text { Large leaking } \\
\text { PMT } \\
\text { Xe afterpulse } \\
>0.1 \%\end{array}$ & $\begin{array}{r}\text { Small leaking } \\
\text { PMT } \\
\text { Xe afterpulse } \\
<0.1 \%\end{array}$ & $\begin{array}{r}\text { Large leak } \\
\text { probability }\end{array}$ \\
\hline R13111 & $5 \mathrm{~N} \mathrm{Al}$ & 58 & 16 & 10 & $28 \pm 6 \%$ \\
\hline R11410 & $5 \mathrm{~N} \mathrm{Al} \mathrm{[15]}$ & 44 & 7 & 1 & $16 \pm 6 \%$ \\
\hline \hline R11410 & $4 \mathrm{~N} \mathrm{Al}$ & 23 & 0 & 1 & $<10 \%$ \\
\hline R11410 & $\begin{array}{l}5 \mathrm{~N} \mathrm{Al} \text { with } \\
\text { special handling }\end{array}$ & 28 & 0 & 0 & $<8 \%$ \\
\hline
\end{tabular}

\section{Conclusion}

We developed a new three-inch low-BG PMT, the Hamamatsu R13111 PMT, which can operate in LXe. 
The R13111 has a convex photocathode that gives it a large angular acceptance and improved timing resolution. For all tested points on the photocathode, relative efficiency multiplied by the quantum efficiency is higher than $70 \%$. The TTS of this new PMT is $2.11_{-0.18}^{+0.31} \mathrm{nsec}$, a large improvement over the $6.87 \pm 0.31 \mathrm{nsec}$ TTS for the R10789. This helps towards more effective pulse shape discrimination.

RIs in the R13111 PMTs manufactured in 2016 are $0.44 \pm 0.06 \mathrm{mBq}$ of ${ }^{226} \mathrm{Ra},{ }^{238} \mathrm{U}$ less than $1.4 \mathrm{mBq}, 0.20 \pm 0.06 \mathrm{mBq}$ of ${ }^{228} \mathrm{Ra}, 2.0 \pm 0.5 \mathrm{mBq}$ of ${ }^{40} \mathrm{~K}$ and $0.13 \pm 0.04 \mathrm{mBq}$ of ${ }^{60} \mathrm{Co}$. The reduction from the $\mathrm{R} 10789$ values is large: factors of 5, 6, 7 and 30 for ${ }^{226} \mathrm{Ra},{ }^{228} \mathrm{Ra},{ }^{40} \mathrm{~K}$, and ${ }^{60} \mathrm{Co}$, respectively. These numbers are the world's best when compared to other currently available PMTs for use in LXe dark matter search experiments.

For long-term stable operation in LXe, we studied the Xe leak problem which was reported by several groups. We identified that the material and treatment of the aluminum seal between the PMT's entrance window and the body causes the Xe leaks. When the seal was changed to highpurity $5 \mathrm{~N}$ aluminum for the R13111 and the R11410 PMT from $98.5 \%$ purity aluminum used for the R10789 PMT, leaks started occurring. From our study, we observed that the problem is solved by handling the $5 \mathrm{~N}$ aluminum seal with sufficient care for the seal's surface during the assembly process.

With its low radioactivity, excellent timing resolution, and large angular acceptance the new R13111 PMT can show highly good performance in future low BG LXe detectors.

\section{Acknowledgments}

We thank Hamamatsu Photonics K. K. for the cooperation in producing the low BG PMTs. We gratefully acknowledge the cooperation of Kamioka Mining and Smelting Company. We also would like to thank OKAMOTO GLASS Co. Ltd. for their help in the glass synthesis. This work was supported by the Japanese Ministry of Education, Culture, Sports, Science and Technology, Grantin-Aid for Scientific Research, ICRR Joint-Usage, JSPS KAKENHI Grant Number, 19GS0204, 26104004, 19H05805, and partially by the National Research Foundation of Korea Grant (NRF2011-220-C00006) and Institute for Basic Science (IBS-R017-G1-2018-a00).

\section{References}

[1] K. Abe et al. [XMASS Collaboration], "XMASS detector," Nucl. Instrum. Meth. A 716 (2013) 78 [arXiv:1301.2815 [physics.ins-det]].

[2] D. S. Akerib et al. [LUX Collaboration], “The Large Underground Xenon (LUX) Experiment," Nucl. Instrum. Meth. A 704 (2013) 111 [arXiv:1211.3788 [physics.ins-det]].

[3] X. Cao et al. [PandaX Collaboration], "PandaX: A Liquid Xenon Dark Matter Experiment at CJPL," Sci. China Phys. Mech. Astron. 57 (2014) 1476 [arXiv:1405.2882 [physics.ins-det]].

[4] E. Aprile et al. [XENON Collaboration], “The XENON1T Dark Matter Experiment,” Eur. Phys. J. C 77 (2017) no.12, 881 [arXiv:1708.07051 [astro-ph.IM]].

[5] T. Suzuki et al. [XMASS Collaboration], "Search for WIMP- ${ }^{129}$ Xe inelastic scattering with particle identification in XMASS-I,” Astropart. Phys. 110, 1 (2019) [arXiv:1809.05358 [astro-ph.CO]]. 
[6] M. Kobayashi et al. [XMASS Collaboration], "Search for sub-GeV dark matter by annual modulation using XMASS-I detector,” Phys. Lett. B 795, 308 (2019) arXiv:1808.06177 [astro-ph.CO].

[7] K. Abe et al. [XMASS Collaboration], "Search for dark matter in the form of hidden photons and axion-like particles in the XMASS detector," Phys. Lett. B 787, 153 (2018) doi:10.1016/j.physletb.2018.10.050 [arXiv:1807.08516 [astro-ph.CO]].

[8] K. Abe et al. [XMASS Collaboration], "A direct dark matter search in XMASS-I," Phys. Lett. B 789, 45 (2019) [arXiv:1804.02180 [astro-ph.CO]].

[9] K. Abe et al. [XMASS Collaboration], "Direct dark matter search by annual modulation with 2.7 years of XMASS-I data," Phys. Rev. D 97, no. 10, 102006 (2018) [arXiv:1801.10096 [astro-ph.CO]].

[10] K. Abe et al., "Light WIMP search in XMASS," Phys. Lett. B 719, 78 (2013) [arXiv:1211.5404 [astro-ph.CO]].

[11] K. Abe et al. [XMASS Collaboration], "Development of low radioactivity photomultiplier tubes for the XMASS-I detector,” Nucl. Instrum. Meth. A 922, 171 (2019) [arXiv:1808.03617 [physics.ins-det]].

[12] B. J. Mount et al., "LUX-ZEPLIN (LZ) Technical Design Report," arXiv:1703.09144 [physics.ins-det].

[13] P. Barrow et al., "Qualification Tests of the R11410-21 Photomultiplier Tubes for the XENON1T Detector”, JINST 12 (2017) P01024 [arXiv:1609.01654v2 [astro-ph.IM]]

[14] S. Li et al. "Performance of photosensors in the PandaX-I experiment", JINST 11 (2016) T02005 [arXiv:1511.06223v3 [physycs.ins-det] ]

[15] E. Aprile et al. [XENON Collaboration], "Lowering the radioactivity of the photomultiplier tubes for the XENON1T dark matter experiment,” Eur. Phys. J. C 75 (2015) 546 [arXiv:1503.07698 [astro-ph.IM]].

[16] S. Agostinelli et al. [GEANT4 Collaboration], "GEANT4: A Simulation toolkit,” Nucl. Instrum. Meth. A 506 (2003) 250.

[17] Eurofins EAG Materials Science Tokyo Corporation, http://www.nanoscience.co.jp/

[18] Toray Research Center, https://www.toray-research.co.jp/

[19] D. S. Akerib et al., "An Ultra-Low Background PMT for Liquid Xenon Detectors," Nucl. Instrum. Meth. A 703, 1 (2013) [arXiv:1205.2272 [physics.ins-det]].

[20] B. Xu [XMASS Collaboration], "XMASS 1.5: The next step in Kamioka, Japan," J. Phys. Conf. Ser. 718, no. 4, 042064 (2016). 